\title{
Euthanasie
}

\section{Der Standpunkt der Schweizerischen Gesellschaft für Palliative Medizin, Pflege und Begleitung (SGPMP)}

\section{F. Stiefel, H. Neuenschwander}

Der Standpunkt der Schweizerischen Gesellschaft für Palliative Medizin, Pflege und Begleitung (SGPMP) zur Euthanasie-Debatte stützt sich auf eine im November 2000 durchgeführte Befragung der Mitglieder [1] und auf eine Diskussion ihrer Ergebnisse im Vorstand der Gesellschaft. Der folgende Text wurde durch eine grosse Mehrheit der Vorstandsmitglieder der SGPMP gutgeheissen.

Die SGPMP ist der Auffassung, dass es verschiedene Argumente für und gegen ärztliche Beihilfe zur Selbsttötung und direkte aktive Sterbehilfe gibt (siehe Definitionen) [2]

Neben ethischen, juristischen und weltanschaulichen Argumenten ist aber von Bedeutung, dass Sterbewünsche bei Schwerkranken sehr oft Ausdruck einer physischen oder psychischen Belastung sind, ausgelöst durch Schmerzen, Atemnot, depressive Verstimmungen oder familiäre Konflikte [3]. Klinische Erfahrungen und wissenschaftliche Untersuchungen zeigen, dass bei einer fachgerechten Behandlung die Wünsche nach direkter aktiver Sterbehilfe oder ärztlicher Beihilfe zur Selbsttötung in der Regel nur vorübergehend bestehen $[4,5]$.

Die SGPMP weist darauf hin, dass Palliative Care (siehe Definitionen) in den letzten Jahrzehnten bedeutende Fortschritte in der klinischen Versorgung von schwerkranken Menschen erreicht hat [6]. Dies hat in verschiedenen Ländern, wie beispielsweise in England oder Australien, zu einem Ausbau von Palliative Care geführt mit Bettenstationen in Akutspitälern, Hospizen, ambulanten Diensten, intra- und extrahospitaliären Konsiliardiensten und palliativer Spitexversorgung. Auch in der Schweiz hat sich Palliative Care in den letzten Jahren etabliert. Eine nationale Bestandesaufnahme (siehe "Palliative Care in der Schweiz») zeigt aber, dass in der Schweiz ein ungenügendes Angebot besteht, und dass es bedeutende interkantonale Unterschiede gibt [7]. Zudem ist der Zugang zu Palliative Care noch hauptsächlich auf Krebskranke beschränkt.

Politische Instanzen, wie der Bundesrat [8] und der Nationalrat [9], Berufsverbände, wie der Schweizerische Berufsverband der Krankenschwestern und Krankenpfleger (SBK), die Verbindung der Schweizer

Korrespondenz:

PD Dr. med. Friedrich Stiefel

CHUV

Service de Psychiatrie de liaison

CH-1011 Lausanne

E-mail: frederic.stiefel@inst. hospvd.ch
Ärztinnen und Ärzte FMH [10] und die Schweizerische Akademie der medizinischen Wissenschaften (SAMW) [11], haben auf die Notwendigkeit eines Ausbaus der Palliative Care in der Schweiz hingewiesen. Die SGPMP erachtet es daher als inkonsequent, die ärztliche Beihilfe zur Selbsttötung und die direkte aktive Sterbehilfe in der Schweiz gesetzlich zu verankern, bevor jeder schwerkranke, bedürftige Mensch Zugang zu qualitätskontrollierter Palliativmedizin hat. Diese Forderung und die entsprechenden Standards für die Qualitätskontrolle der klinischen Versorgung [12] und Empfehlungen für die Ausbildung in Palliative Care [13] wurden an einer Nationalen Konsensustagung am 1. Februar 2001 verabschiedet (siehe "Freiburger Manifest») [14].

Bevor in der Schweiz kein vollständiger Zugang zu Palliative Care besteht, sollte eine gesetzliche Verankerung der ärztlichen Beihilfe zur Selbsttötung und der direkten aktiven Sterbehilfe nicht angestrebt werden. Dagegen wünscht die SGPMP, dass das Angebot von Palliative Care ausgebaut wird und die öffentliche Diskussion über die "End of Life Care» fortgesetzt wird.

\section{Literatur}

1 Neuenschwander H, Bittel N und Stiefel F. Stichwort «Euthanasie» - Was denken die Mitglieder der Schweizerischen Gesellschaft für Palliative Medizin, Pflege und Begleitung? Infokara 2001; Vol. 2.

2 Sterbehilfe - Bericht der Arbeitsgruppe an das Eidgenössische Justiz- und Polizeidepartement. März, 1999.

3 Stiefel F. Sterbewunsch, Suizidgedanken, Suizid und aktive Euthanasie bei Krebskranken mit fortgeschrittenem Leiden. In: Mettner M (Hrsg.). Wie menschenwürdig sterben? Zürich: NZN Buchverlag AG; 2000. S. 29-36.

4 Owen C, Tennat C, Levi J, Jones M. Suicide and euthanasia: patient attitudes in the context of cancer. Psycho-Oncology 1992;1:79-88.

5 Guex P. La maladie terminale. Dans: Guex P (éd.). Psychologie et Cancer. Manuel de psycho-oncologie. Lausanne: Editions Payot; 1989. p. 131-51.

6 Neuenschwander H, Steiner N, Stiefel F, de Stoutz N, Humbert N, St.-Laurent-Gagnon T. Palliativmedizin auf einen Blick - Ein Handbuch für Ärztinnen und Ärzte. Schweizerische Krebsliga: 2000.

7 Raemy-Bass C, Lugon JP, Eggimann JC. Bestandesaufnahme der Palliative Care in der Schweiz 1999/2000. Schweizerische Krebsliga und Schweizerische Gesellschaft für Palliative Medizin, Pflege und Begleitung: 2001.

8 Passive Sterbehilfe und indirekte aktive Sterbehilfe / Bundesrat verabschiedet Bericht zum Postulat Ruffy. Pressemitteilung 5. Juli 2000.

9 Motion Rossini im Nationalrat vom 22. Juni 2000: Finanzierung der Palliativbehandlung.

10 Pflege und Behandlung in der Endphase des Lebens. Eine gemeinsame Erklärung des Schweizerischen Berufsverbands der Krankenschwestern und Krankenpfleger (SBK) und der Verbindung der Schweizer Ärztinnen und Ärzte FMH. Bern: 2001.

11 Medizinisch-ethische Richtlinien für die ärztliche Betreuung sterbender und zerebral schwerst geschädigter Patienten. Schweizerische Akademie der Medizinischen Wissenschaften (SAMW): 1995.

12 Eychmüller S, Knipping C, Lang M, Mazzocato C, TschoppHafenbrack A, Zlatiev-Scocard N. Standards: Grundsätze und Richtlinien für Palliative Medizin, Pflege und Begleitung in der Schweiz. Schweizerische Gesellschaft für Palliative Medizin, Pflege und Begleitung: 2001. 
13 Porchet F, Eychmüller S, Steiner N. Aus- und Weiterbildung in "Palliative Care». Entwurf für nationale Empfehlungen. Schweizerische Gesellschaft für Palliative Medizin, Pflege und Begleitung: 2001
14 Eychmüller S, Porchet F, Stiefel F, von Wyss M. Das Freiburger Manifest: Eine nationale Strategie für die Entwicklung von Palliative Care in der Schweiz. Schweizerische Gesellschaft für Palliative Medizin, Pflege und Begleitung: 2001.

\section{Definitionen}

Palliative Care, Definition

Die palliative Medizin, Pflege und Begleitung umfasst alle medizinischen Behandlungen, die pflegerischen Interventionen sowie die psychische, soziale und geistige Unterstützung kranker Menschen, die an einer progredienten, unheilbaren Erkrankung leiden. Ihr Ziel besteht darin, Leiden zu lindern und die bestmögliche Lebensqualität des Kranken und seiner Angehörigen zu sichern (Schweizerische Gesellschaft für Palliative Medizin, Pflege und Begleitung. Statuten 2000, Art. 3).

\section{Palliative Care, Leistungen}

Palliative Care wird von einem interdisziplinären Team angeboten mit dem Ziel, körperliches Wohlbefinden der Patienten zu erhalten, ihr psychisches Leiden $\mathrm{zu}$ lindern, ihre sozialen Beziehungen $\mathrm{zu}$ unterstützen, und sie ihren persönlichen Glauben leben zu lassen. Die Behandlungen von Palliative Care werden nie in der Absicht gegeben, den Tod herbeizuführen; es werden aber auch keine Massnahmen getroffen, um das Leben um jeden Preis zu verlängern. "Palliative Betreuung - aus Verantwortung für schwerkranke Menschen", Schweizerische Gesellschaft für Palliative Medizin, Pflege und Begleitung (Hrsg) 2001.

\section{Die Schweizerische Gesellschaft für Palliative Medizin, Pflege und Begleitung (SGPMP)}

Die SGPMP vereinigt über 1000 in Palliative Care tätige Mitglieder; sie ist politisch und konfessionell neutral (Statuten 2000, Art. 6). Weitere Informationen können bei dem Präsidenten der SGPMP bestellt werden: PD Dr. med. F. Stiefel, Service de Psychiatrie de Liaison, CHUV, 1011 Lausanne.

\section{Passive Sterbehilfe}

Verzicht auf die Aufnahme oder Abbruch von lebenserhaltenden Massnahmen. Diese Form der Sterbehilfe ist gesetzlich nicht ausdrücklich geregelt, wird aber als erlaubt angesehen. Sterbehilfe Bericht der Arbeitsgruppe an das Eidgenössische Justiz- und Polizeidepartement, März, 1999.

\section{Indirekte aktive Sterbehilfe}

Die zur Linderung des Leidens eingesetzten Mittel setzen als Nebenwirkung die Lebensdauer herab. Diese Art der Sterbehilfe ist im Strafgesetzbuch nicht ausdrücklich geregelt, gilt aber als grundsätzlich erlaubt. Sterbehilfe - Bericht der Arbeitsgruppe an das Eidgenössische Justiz- und Polizeidepartement, März, 1999.
Ärztliche Beihilfe zur Selbsttötung

Selbsttötung mit Hilfe eines Arztes. Die Beihilfe zur Selbsttötung ist in der Schweiz nicht strafbar, sofern ein egoistisches Motiv ausgeschlossen werden kann (Art. 115 des Strafgesetzbuches). Ärztliche Beihilfe zur Selbsttötung wird von Organisationen wie Exit praktiziert, ist aber gemäss den Richtlinien der Schweizerischen Akademie der medizinischen Wissenschaften (SAMW) «nicht Teil der ärztlichen Tätigkeit».

\section{Direkte aktive Sterbehilfe}

oft auch einfach "Euthanasie» genannt, ist die Tötung eines Menschen zur Verkürzung seines Leidens. Direkte aktive Euthanasie ist strafbar (Art. 111, 113, 114 des Strafgesetzbuches).

\section{Palliative Care in der Schweiz}

Eine nationale Bestandesaufnahme zur Versorgung mit Palliative Care - durchgeführt im Auftrag der Schweizerischen Krebsliga und der Schweizerischen Gesellschaft für Palliative Medizin, Pflege und Begleitung - kam zu dem Ergebnis, dass in der Schweiz bedeutende interkantonale Unterschiede bestehen, dass Palliative Care noch stark auf urbane Zentren konzentriert ist, und dass vor allem Krebskranke Zugang zu Palliative Care haben. Patienten mit anderen Erkrankungen, Kinder und ältere Menschen können nicht von den Leistungen der Palliative Care profitieren. Erschwert wird die Verbreitung der Palliative Care durch den Umstand, dass bis anhin nur wenige Ausbildungsmöglichkeiten in der Schweiz bestehen und eine anerkannte Qualitätskontrolle erst im Entstehen ist.

\section{Freiburger Manifest}

In dem am 1. Februar 2001 in Freiburg von über 550 im Gesundheitswesen tätigen Personen unterstützten Manifest werden verschiedene Massnahmen zur Entwicklung von Palliative Care gefordert. Dazu gehören Massnahmen, die auf Bundesebene (wie Aufnahme von Palliative Care in die medizinische und pflegerische Ausbildung und in den Pflichtleistungskatalog des KVGs) und auf kantonaler Ebene getroffen werden (regionale Bedarfsanalyse und Umwandlung bestehender Strukturen in ambulante und stationäre Palliative-Care-Dienste). Das Freiburger Manifest sowie die Zusammenfassung der nationalen Bestandesaufnahme «Palliative Care in der Schweiz» kann beim Präsidenten der Schweizerischen Gesellschaft für Palliative Medizin, Pflege und Begleitung bestellt werden. 